\title{
Structural Elements Contributing to Efficient -1 Ribosomal Frameshifting in BWY V Pseudoknot
}

\author{
So Jung Park, Hee Jung Park, Sangho Lee, and Yang-Gỵun Kim \\ Departments of Chemistry and Biological Science, School of Katural Sciences, Sungkumkwan Lniversity, \\ Sumon +40-746. Korea. "E-mail: vghimmitiáskhi.edu \\ Received Jantiary 12, 2009, Accepted Jamary 28, 2009
}

Key Words: Ribosomal frameshifting, RNA pseudoknot, BWYV, SRV-1

The programmed -1 ribosomal frameshifting (-lRFS). one of the programmed altemative reading mechanisms in translation. moves tRNAs inside ribosome toward -1 direction during translation at a predetermined sequence on an mRNA with a certain frequency. production of two gene products from a single mRNA. This unusual mechanism is wide-spread among retroviruses. plant viruses. retrotransposons. bacteria and yeast. Two cis-acting elements in mRNA are generally required to stimulate efficient -IRFS. ${ }^{1,2}$ One is the slippery sequence consisting of a hepta-

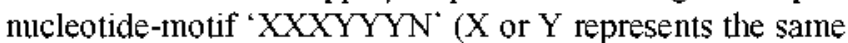
base, and $\mathrm{N}$ is any) where -1 frameshifting of tRNAs actually occurs. The other element is an RNA stnicture. mostly RNA pseudoknots, locating 4-8 nucleotides downstream of the slippery sequence.

Many studies have correlated the stability of an RNA stnicture. notably RNA pseudoknots, to the high efficiency of -IRFS ${ }^{-18}$ Frameshifting-stimulating pseudoknots referred to as pseudoknots unless specified otherwise. exhibit classic $\mathrm{H}$-type pseudoknot-folds with two stacked stems (S1 and S2) and two loops (L1 and L2) (Fig. 1A). Structural elements responsible for the stability and the integrity of the psendoknots include quasi-continuous base-stacking in S1 and S2 and an extensive Sl-L2 triplex interaction.

Structural diversity among pseudoknots appears to be one of major culprits for various viral systems to achieve different -IRFS efficiencies optimized to their own needs. The pselldoknots in BWYV (beet western yellow virus) and SRV-1 (simian retrovinis-1) provide an interesting instance for the stnictural diversity. BWYV and other luteoviral pseudoknots. which mediate P1-P2 production through - IRFS. are relatively small and tightly folded with a characteristically very short \$2 ( 3 base pairs) (Fig. 1A). These luteoviral pseudoknots produce considerable degrees of -IRFS and show a strong sequence preference in both L 1 and L2 for efficient -IRFS. From the structure-function analy sis of the luteoviral pseudoknots. ${ }^{5,9-1+}$ it is well established that tertiary interactions are crucial: in particular. C8 in the L 1 of the BWYV pseudoknot engages with GI2, A25 and C26 to form the complex quadnuple-base interaction. In contrast, SRV-1 pseudoknot, located at gag-pro junction contains a relatively long $\$ 2$ ( 5 base pairs without counting the A-U base pair in the stem junction) (Fig. IA). Unlike the loops in the BWYV pseudoknot. the length and the base identity of loops in the SRV-1 pseudoknot are rather insignificant. It has been shown that even large deletion of L2 can be tolerable for efficient -lRFS. ${ }^{81:}$

Structural comparison suggests that the stems of the pseudoknots of BWYV and SRV-I employ different mechanisms to ensure the stability of the pseudoknots. The SRV-1 pseudoknot takes advantage of intrinsically high stem stability provided by the long $\mathrm{S} 2$ stem for efficient -IRFS. In contrast. the BWYV and other luteoviral pseudoknots possess tightly folded structures through the quadruple-base interaction between $\mathrm{S} 2-\mathrm{L} 1$ as well as the triplex interactions between SI-L2, which are enough to compensate for low stem stability caused by the short S2 stem. ${ }^{12.16}$ Thus. it is of great interest to transforn the BWYV pseudoknot into SRV-l-like pseudoknot

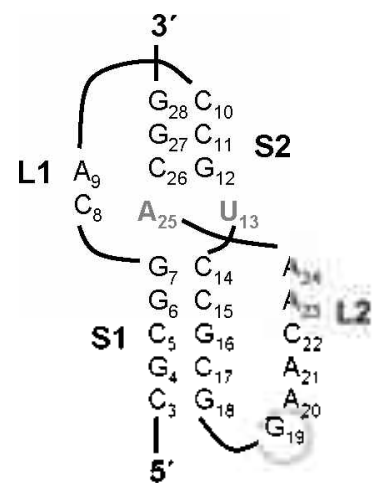

BWYV (10.8\%)

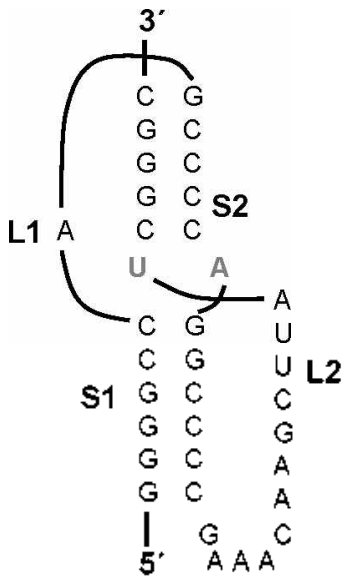

SRV-1 (12.7\%)

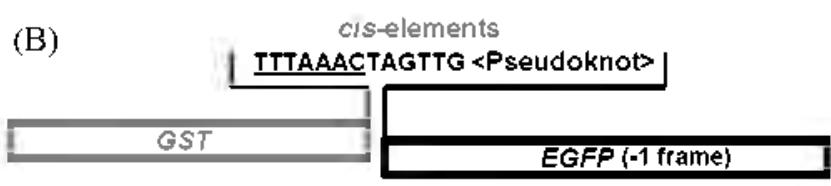

GST product by normal translation (9 methionines)

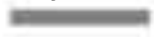

GSTEGFP fusian praduct by -1 frameshifting (14 methionines)

Figure 1. (A) Secondary-structure diagrams of the BWYV and SRV-1 pseudoknots. Numbers in parentheses indicate - IRFS efficiency detemined using in vitro sy stem. (B) Schematic representation of the reporter construct and resulting protein products used for in vitro-IRFS assay, Boxed regions indicate ORFs for GST (Grey) and $E G F P$ (Black). 
Table 1. Sequence and-1RFS efficiency of the wild-type and SRV-1-like mutants of the BWYV psendoknot

\begin{tabular}{|c|c|c|c|c|c|c|c|c|}
\hline \multirow[t]{2}{*}{ Name } & \multicolumn{7}{|c|}{ Pseudoknot Sequence } & \multirow[t]{2}{*}{ Effïiency $(\%$} \\
\hline & Sl & $\mathrm{Ll}$ & $\$ 2$ & $\mathbf{J}$ & Sl & $\mathrm{L} 2$ & $\$ 2$ & \\
\hline $\mathrm{WT}^{a}$ & CGCGG & $\mathrm{CA}$ & $\mathrm{CCG}$ & $\mathbf{U}$ & $\mathrm{CCGCG}$ & GAACAA & A CGG & 10.8 \\
\hline $\mathrm{mS}-\mathrm{B}$ & CGCGG & $\mathrm{CA}$ & $\mathrm{GCCCG}$ & $\mathbf{U}$ & $\mathrm{CCGCG}$ & GAACAA & A CGGGC & 8.4 \\
\hline $\mathrm{mS}-\mathrm{B}: \mathrm{C} 8 \mathrm{~A}$ & $\mathrm{CGCGG}$ & $\mathrm{AA}$ & $\mathrm{GCCCO}$ & $\mathbf{U}$ & $\mathrm{CCGCG}$ & GAACAA & A $C G G G C$ & 5.0 \\
\hline $\mathrm{mS}-\mathrm{B}: \mathrm{A} 25 \mathrm{G}$ & $\mathrm{CGCGG}$ & $\mathrm{CA}$ & $\mathrm{GCCCG}$ & $\mathbf{U}$ & $\mathrm{CCGCG}$ & GAACAA & G CGGGC & 0.8 \\
\hline mSL-B & CGCGG & $\mathrm{A}$ & $\mathrm{GCCCG}$ & $\mathbf{U}$ & $\mathrm{CCGCG}$ & $\overline{\text { GAACAA }}$ & A CGGGC & 4.4 \\
\hline mSL-B:A9C & $\mathrm{CGCGG}$ & C & $\mathrm{GCCCG}$ & $\mathbf{U}$ & $\mathrm{CCGCG}$ & GAACAA & A CGGGC & 7.4 \\
\hline mSL-B:A9G & CGCGG & $\underline{G}$ & $\mathrm{GCCCG}$ & $\mathbf{U}$ & $\mathrm{CCGCG}$ & GAACAA & A CGGGC & 2.3 \\
\hline mSL-B:A9U & CGCGG & $\overline{\mathrm{U}}$ & $\mathrm{GCCCG}$ & $\mathbf{U}$ & $\mathrm{CCGCG}$ & $\overline{\mathrm{GAACAA}}$ & A CGGGC & 4.4 \\
\hline MSL,I-B & $\mathrm{CGCGG}$ & A & $\mathrm{GCCCG}$ & $\mathbf{A}$ & $\mathrm{CCGCG}$ & GAACAA & U CGGGC & 6.1 \\
\hline mS-B:UC 19 & CGCGG & $\mathrm{CA}$ & $\mathrm{GCCCG}$ & $\mathbf{U}$ & $\mathrm{CCGCG}$ & UCAACAA & A CGGGC & 26.8 \\
\hline $\mathrm{mS}-\mathrm{B}: \mathrm{GC} 19$ & CGCGG & $\mathrm{CA}$ & $\mathrm{GCCCG}$ & $\mathbf{U}$ & $\mathrm{CCGCG}$ & GCAACAA & A CGGGC & 10.2 \\
\hline mSL-B:UC19 & $\mathrm{CGCGG}$ & A & $\mathrm{GCCCO}$ & $\mathbf{U}$ & $\mathrm{CCGCG}$ & UCAACAA & A $C G G G C$ & 12.1 \\
\hline mSL-B:GC 19 & $\mathrm{CGCGG}$ & $\mathrm{A}$ & $\mathrm{GCCCG}$ & $\mathbf{U}$ & $\mathrm{CCGCG}$ & $\overline{\text { GCAACAA }}$ & A CGGGC & 6.8 \\
\hline WT:UC 19 & CGCGG & $\mathrm{CA}$ & $\mathrm{CCG}$ & $\mathbf{U}$ & $\mathrm{CCGCG}$ & UCAACAA & A CGG & $30.5^{b}$ \\
\hline WT:GC19 & $\mathrm{CGCGG}$ & $\mathrm{CA}$ & $\mathrm{CCO}$ & $\mathbf{U}$ & $\mathrm{CCGCG}$ & GCAACAA & A $C G G$ & $13.3^{4}$ \\
\hline
\end{tabular}

Stems (SI, S2), loops ( L I, L2) and junctions ( J) between stems and loops are indicated above pseudoknot sequences. The -lRFS efficiency of the wild-type BWYV pseudoknot, $10.8 \%$, was used to nomalize -IRFS efficiency of each pseudoknot. "Wild-type BWYV pseudoknot. ${ }^{b}$ These results were published previously in the article by Kin et al ${ }^{s}$

to investigate the contributions of the stem stability and the quadruple-base interactions to the efficient -IRFS. Here we examined the effects of the stnictural components of the BWYV pseudoknot on the -lRFS efficiency by altering the length and sequences of the $\$ 2$ and the $L 1$ to closely resemble the SRV-1 pseudoknot.

To study the effect of the length change in S2 on the - IRFS efficiency of the BWYV pseudoknot. we added two base pairs at the top side to the S2 of the BWYV pseudoknot. which resulted in a mutant pseudoknot containing five base-pair $\$ 2$. referred to as $\mathrm{mS}-\mathrm{B}$ (Fig. 2A. left panel). This extended S2 in $\mathrm{mS}-\mathrm{B}$ is expected to provide more stability to the pseudoknot. However it is likely to cause perturbation of the quadruple-base interactions between $\mathrm{L} 1$ and $\mathrm{S} 2$ as well. Various mutations on $\mathrm{mS}-\mathrm{B}$ were introduced (Table $\mathrm{I}$ ) and their -IRFS efficiencies determined by in vitro translation assay. Plasmids for the in vitro assay have two genes in tandem. gluthathione $S$-transferase $(G S T)$ followed by enhanced green fluorescent protein
( $E G F P)$. These two genes are connected by -l frame. Accordingly the downstrean $E G F P$ gene would be translated as a fusion protein product (GST-EGFP) with the upstream GST gene only when -lRFS occurs during the translation (Fig. 1B). All pseudoknot-forming sequences were inserted after the slippery sequence with the 6-base spacer.

Results from the in vitro translational assay showed that -IRFS efficiency of $\mathrm{mS}-\mathrm{B}$ was reduced to $c a$. $80 \%$ level of that of the BWYV wild type (see Fig. 1A, left panel and Fig. $2 \mathrm{~A}$. left panel). The two mutations at mucleotides participating the quadruple-base interaction. $\mathrm{mS}-\mathrm{B}: \mathrm{C} 8 \mathrm{~A}$ and $\mathrm{mS}-\mathrm{B}: \mathrm{A} 25 \mathrm{G}$, decreased-IRFS efficiencies even further (Fig. 2A. left panel). In particular. -IRFS was almost abolished by the A25G mutation in mS-B as sinularly seen in the same nutation in the wild-type BWYV pseudoknot. These observations suggest that the quadruple-base interaction may still play a role in the stabilization of $\mathrm{mS}-\mathrm{B}$.

Next we modified mS-B by reducing $\mathrm{Ll}$ to one nucleotide
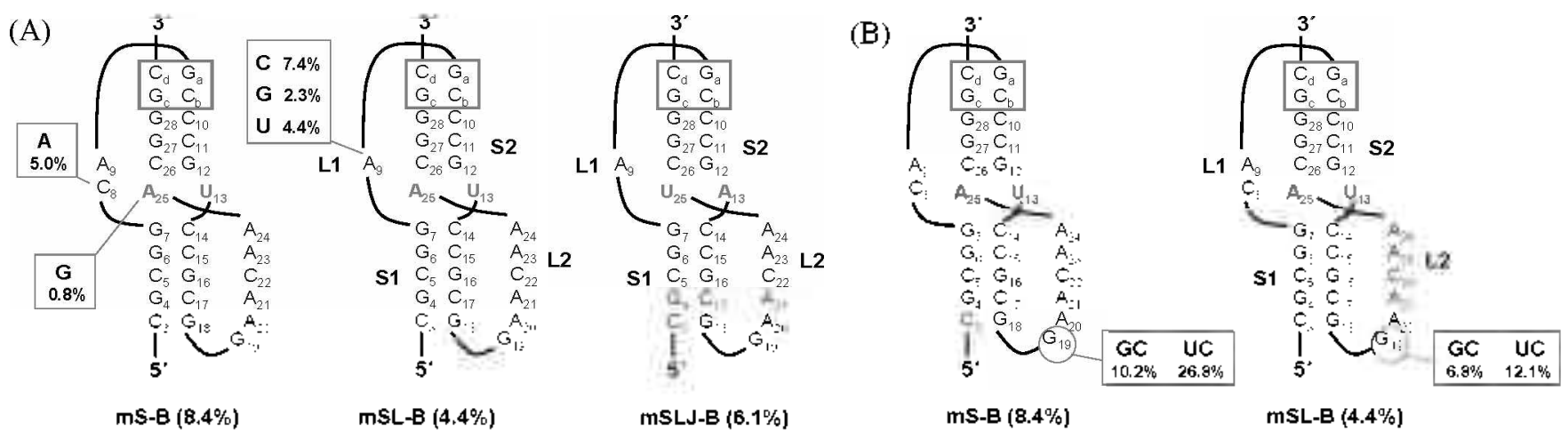

Figure 2. Constructs of the BWYV psendoknot minicking the SRV-l pseudoknot and their effects on -lRFS efficiency. The -1 RFS efficiency of the wild-ty pe BWYV pseudoknot in this study is $10.8 \%$. The numbers near the mutations correspond to the - 1 RFS efficiency. (A) SR V-I-like pseudoknot constructs (mS-B, mSL-B and mSL.T-B) and their mutants. (B) Mutations at the SI-L2 linker region of mS-B and mSL-B. 
and inverting the junctional base pair (from $\mathrm{A} 25: \mathrm{U} 13$ to U13:A25). These constnicts. mSL-B and mSLJ-B, respectively. mimic the SRV-I pseudoknot by possessing the extended S2 ( 5 base pairs) and a shortened L 1 (one nucleotide) (Fig. $2 \mathrm{~A}$. center and right panels). mSL-B exhibited reduced - IRFS efficiency $(4.4 \%)$ to an half or less than $\mathrm{mS}-\mathrm{B}(8.4 \%)$ and the BWYV wild type $(10.8 \%)$ while mSLJ-B showed higher -1 RFS efficiency (6.1\%) than mSL-B (4.4\%) (Fig. 2A). The results indicate that the disruption of the quadnuple-base interaction by shortening $\mathrm{Ll}$ can be compensated to some extent by the inverted junctional U25:A13 pair.

Unlike BWYV pseudoknot as reported previously , there is no marked sequence preference exhibited in the Ll of SRV-l pseudoknot. ${ }^{8}$ Accordingly. we investigated the sequence preference of the shortened L 1 in mSL-B and compared with that in the wild-type BWYV pseudoknot. We examined the - IRFS efficiencies of all four nucleotides in the $\mathrm{Ll}$ of $\mathrm{mSL}-\mathrm{B}$ (Fig. 2A). mSL-B with the cy tosine in the Ll (mSL-B:A9C) showed the highest -IRFS efficiency (7.3\%) among the four nucleotides tested. which is about $70 \%$ of that of the BWYV wild type $(10.8 \%)$. mSL-B:A9U showed the same -IRFS efficiency $(4.4 \%)$ as mSL-B while mSL-B:A9G exhibited the reduced - LRFS efficiency to $2.3 \%$. The highest - IRFS efficiency by mSL-B:A9C implies that mSL-B may not completely discard the quadruple-base interaction in which the identity of cy tosine in the $\mathrm{Ll}$ is critical for the efficient - IRFS. This cytosine preference suggests the possible formation of a quadruple-base interaction in mSL-B although its formation may not be as strong as in the wild-type BWYV pseudoknot.

Finally we performed mutational analysis of the S1-L2 linker region. Previously a drastic increase of -lRFS efficiency in mutations at G19 in the S1-L2 linker region was noted. seemingly irrespective of structure-stabilizing effect (Table 1). It was postulated that G19 might contact a part of ribosome during the -lRFS event. which in turn may stimulate -IRFS through intermolecular interaction(s). To examine the effects of G19 on the -IRFS efficiencies in our SRV-1-like pseudoknots. we mutated G19 of the S1-L2 linker region to G19C19a and U19C19a by insertion and substitution in both mS-B and mSL-B (Fig. 2B). Although it does not appear that the changes of the SI-L2 linker region are major factors for integrity of BWYV pseudoknot. the increasing effect of these mutations on -lRFS efficiency was similarly reproduced in both $\mathrm{mS}-\mathrm{B}$ and $\mathrm{mSL}-\mathrm{B}$ (Fig. $2 \mathrm{~B}$ ) as seen in the wild-type BWYV pseudoknot (Table 1. WT:UC19 and WT:GC19). In addition. the identities of the bases at the S1-L2 linker region also affected the -IRFS efficiency greatly. Specifically G to U substitution was substantially more effective on promoting -IRFS and further enhancing in accompanying with $C$ insertion.

Overall. transforming the BWYV pseudoknot into the SRV-1-like pseudoknot (mS-B) by increasing the length of S2 resulted in a bit weaker but still robust frameshifting pseudoknot. The extended $\mathrm{S} 2$ in $\mathrm{mS}-\mathrm{B}$ (from 3 base-pairs to 5 base-pairs) might lead to almost complete loss or significant reduction of the quadniple-base interaction in the L1-\$2 junction due to the base substitution and misalignment of the interacting bases. However the enhanced stability of $\mathrm{mS}-\mathrm{B}$ by having two extra base pairs appears to compensate for the weakened major interactions in the wild-type BWYV pseudoknot. at least considerably if not completely. Moreover. like BWYV pseudoknot, unique sequence preference of the S1-L2 linker region unrelated to the pseudoknot stability was still observed. Thus. our results support that the SI-L2 linker region could increase the -lRFS efficiency by unknown trans-interactions with other component(s) of translational macluinery.

\section{Experimental Section}

Plasmid construction. Plasnids used for in vitro translation assay were constnucted by essentially the same methods described previously. ${ }^{5}$ In brief, the glutathione $S$-transferase (GST) gene and the enhanced green fluorescent protein (EGFP) gene downstream of the GST gene were inserted into the pGEM-3Z vector (Promega). respectively. Various pseudoknot sequences were inserted downstream of the UUUAAAC slippery (Fig. 1B). The resulting constructs contain the two genes connected in out-of-frame. Thus. GST-EGFP fusion protein is translated only when -1RFS occurs at the slippery site.

in vitro frameshifting assay. The $\mathrm{T}_{\mathrm{N}} \mathrm{T} \mathrm{T} 7$-coupled transcription/translation system (Promega) was used according to the manufacturer's protocol as previously described. ${ }^{5}$ To calculate-IRFS efficiency. the ${ }^{35} \mathrm{~S}$-Met labeled samples were run on $12 \%$ SDS polyacry lanuide gels to separate translational products. PhosphorImager (Molecular Dýnamics) was employed to quantify signals of frameshifting ( $1+$ methionines) and non-framslifting (9 methionines) products. As Frameshifting efficiencies were calculated with the formula (I[FS]/14)/ $[(\mathrm{I}[\mathrm{FS}] / 14)+(\mathrm{I}[\mathrm{NFS}] / 9)]$. where $\mathrm{I}[\mathrm{FS}]$ is the signal intensity of the frameshifting product and INFS], the signal intensity of the nonframeshifting product. All individual in vitro assays were accompanied by the wild-type BWYV pseudoknot. Assays were repeated three times or more to determine the average - IRFS efficiencies for all constructs. The -lRFS efficiency of the wild-type BWYV pseudoknot. $10.8 \%$ as reported previously. "was used to normalize-IRFS efficiency of each pseudoknot.

Acknowledgments. This work was supported by the Korea Research Foundation Grant funded by the Korean Government (MOEHRD) (KRF-2005-070-C00106).

\section{References}

1. Gesteland, R. F.; Atkins, J. F. Annt. Rev. Biochent. 1996, 65, 741 .

2. Farabangh, P. T. Aficrobiol. Rev. 1996, 60, 103 .

3. ten Dam, E. B.: Pleij, C. W.; Bosch, L. Thins Genes 1990, 4, 121.

4. Kollmus, $\mathrm{H}$; Hentze, M. W.; Hauser, H. RN4 1996, 2, 316.

5. Kim, Y. G.; Su, L.; Maas, S.; O'Neill, A.; Rich, A. Proc. Natl. Acad. Sci. LSA 1999, 96, 14234.

6. Kollmus, H.; Honigman, A.: Panet, A.: Hauser, H. J. Tinol. 1994. 68,6087

7. Giedroc, D. P.; Theimer, C. A.; Nixon, P. L. J. Nol. Biol. 2000, 298, 167 .

8. ten Dam, E. B.; Verlaan, P. W: Pleij, C. W. R.A 1995, $I, 146$.

9. Kim, Y. G.; Maas, S.: Wang, S. C.: Rich, A. Rt $2000,6,1157$.

10. Connish, P. V.: Hennig, M.; Giedroc, D. P. Proc Natl Acad. Sci. LSA 2005, 102, 12694. 
11. Conish, P. V.: Stammler, S. N.; Giedroc, D. P. RVA 2006, I2, 1959.

12. Nixon, P. L.; Comish, P. V.: Suram, S. V.; Giedroc, D. P. Biochemistry 2002, 11,10665

13. Nixon, P. L.: Giedroc, D. P. J. Mol Biol 2000, 296,659.

14. Nixon, P. L.; Rangan, A.; Kim, Y. G.: Rich, A.: Hoffnan, D. W.;
Hennig, M.; Giedroc, D. P. J. Mol. Biol. 2002, 322,621.

15. ten Dam, E.; Brierley, I.; Inglis, S.; Pleij, C. Nucleic Acids Res. 1994, 22, 2304

16. Pallan, P. S.; Marshall, W. S.; Harp, J.: Jewett, F. C., 3rd; Wawrzak, Z.: Brown, B. A., 2nd, Rich, A.: Egli, M. Biochemistry $2005,4+11315$. 